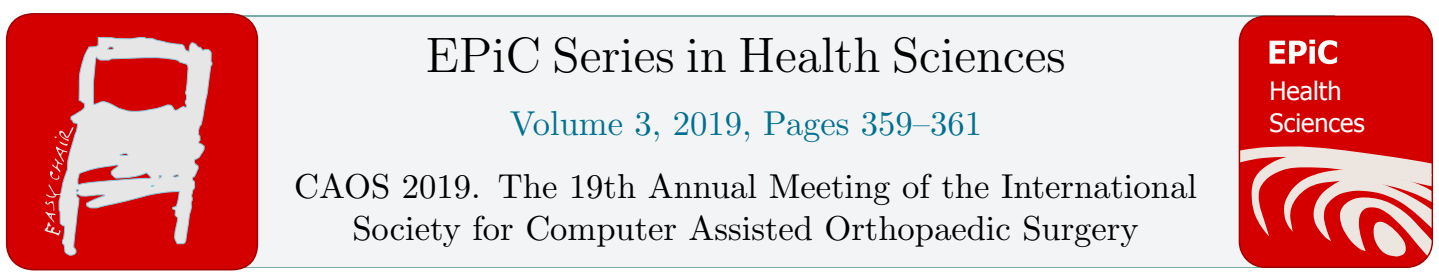

\title{
Comparison of Clinical Outcomes after Total Hip Arthroplasty with and without Computed Tomography-Based Navigation System for Osteonecrosis of the Femoral Head: A Propensity Score Matched Analysis
}

\author{
Kazuma Takashima ${ }^{1 *}$, Takashi Sakai ${ }^{1,2 \dagger}$, Hidetoshi Hamada ${ }^{1}$, \\ Wataru Ando ${ }^{1}$, Masaki Takao ${ }^{1}$, and Nobuhiko Sugano ${ }^{1}$ \\ ${ }^{1}$ Osaka University Graduate School of Medicine, Osaka, Japan \\ ${ }^{2}$ Yamaguchi University Graduate School of Medicine, Yamaguchi, Japan \\ t.kazuma@ hotmail.co.jp, cozy@yamaguchi-u.ac.jp
}

\begin{abstract}
This study aimed to investigate whether use of a computed tomography (CT)-based navigation system reduce the risk of dislocation after total hip arthroplasty (THA) in patients with osteonecrosis of the femoral head (ONFH). A total of 271 hips from 192 consecutive patients that underwent primary THA for ONFH were included. There were 110 hips in non-navigation group, and 161 hips in navigation group. After applying exclusion criteria, 209 hips from 149 patients were selected for this study. Clinical outcomes and complication rates were evaluated, and implant alignments were also calculated. To identify whether the navigation system was useful to prevent dislocation, the inverse probability of treatment weighted Cox regression analysis using a propensity score in relationship to sex, age at surgery, body mass index, and femoral head size was performed. No significant difference was observed in clinical scores between both groups. Dislocation was significantly lower in the navigation group (3 hips, $2.7 \%$ ) than in the non-navigation group (11 hips, $11.2 \% ; \mathrm{p}=0.012$ ), whereas periprosthetic joint infection and aseptic loosening did not differ between the groups. Variance of cup angle was smaller in the navigation group than in the non-navigation group ( $<<0.001)$. Use of the CTbased navigation system (HR; $0.26,95 \% \mathrm{CI}, 0.07-0.98 ; \mathrm{p}=0.047)$ turned out to be the predictor for preventing dislocation. In conclusion, use of the CT-based navigation system provided a precise placement of components, and thus help prevent dislocation in patients with ONFH in the propensity score analysis.
\end{abstract}

* Created the first draft of this document

${ }^{\dagger}$ Masterminded EasyChair and created the first stable version of this document 


\section{Introduction}

Although several studies reported the usefulness of the computed tomography (CT)-based navigation system in THA, there have been no reports that compared the clinical and radiological outcomes of THA performed with or without a CT-based navigation system in patients with ONFH. Therefore, the purpose of this study is to investigate the clinical and radiological outcomes following navigated or non-navigated THA in patients with ONFH.

\section{Materials and Methods}

The Between January 1990 and December 2014, 271 hips from 192 consecutive patients who underwent primary THA for ONFH with a follow-up period of longer than 2 years (mean, 10 years; range, 2-26 years) were included in this study. Of the 271 hips, THA was performed without a CTbased navigation system in 110 hips between January 1990 and February 2004 (non-navigation group), whereas 161 hips underwent THA with a CT-based navigation system (CT-Hip System, Stryker) between March 2004 and December 2014 (navigation group). After applying exclusion criteria, 209 hips (149 patients) that underwent THA through a posterolateral approach were available for this study. Fifty-two hips of men and 46 hips of women (mean age, $47.5 \pm 13.5$ years; mean body mass index (BMI), $23.0 \pm 3.6 \mathrm{~kg} / \mathrm{m} 2$ ) were included in the non-navigation group; 49 hips of men and 62 hips of women (mean age, $50.0 \pm 16.1$ years; mean BMI, $22.4 \pm 3.8 \mathrm{~kg} / \mathrm{m} 2$ ) were included in the navigation group. The average follow-up period in the non-navigation and navigation groups was 15 years (range, $2-26$ years) and 6 years (range, 2-13 years), respectively. We evaluated clinical and radiological outcomes, and also calculated the cup alignment observed in the early postoperative, anteroposterior radiographs. Complications including dislocation, periprosthetic joint infection, and periprosthetic fracture were evaluated. To identify whether the navigation system was useful to prevent dislocation, the inverse probability of treatment weighted (IPTW) Cox regression analysis using a propensity score in relationship to sex, age at surgery, BMI, and femoral head size was performed.

\section{Results}

No significant differences were observed in preoperative and postoperative clinical scores between both groups. Dislocation rates were significantly lower in the navigation group (3 hips, $2.7 \%$ ) than in the non-navigation group (11 hips, $11.2 \% ; \mathrm{p}=0.012)$, although periprosthetic joint infection and periprosthetic fracture did not differ between the groups. Based on the results from the IPTW Cox regression analysis, use of the CT-based navigation system (HR; 0.26, 95\% CI, 0.07-0.98; p = 0.047) turned out to be the predictors for preventing dislocation. Mean cup inclination and anteversion angles in the non-navigation group were $43.2 \pm 5.7^{\circ}$ and $15.5 \pm 6.6^{\circ}$, respectively, and $39.7 \pm 3.0^{\circ}$ and $14.0 \pm$ $2.7^{\circ}$ in the navigation group, respectively. Variance of cup angle was smaller in the navigation group than in the non-navigation group $(\mathrm{p}<0.001)$. The survival rate with revision as the end point at six years of follow-up in the non-navigation group was 97.8\% (95\% CI, 94.7-100\%), and that of the navigation group was $98.2 \%$ (95\% CI, 95.7-100\%); no significant differences were observed between both groups $(\mathrm{p}=0.874)$.

\section{Discussion}

Several studies have demonstrated that the use of a navigation system provided a more precise placement of components than conventional manual techniques, thus eliminating implant malpositioning and the risk of subsequent complications. However, there have been no reports that compare the clinical outcomes and complications of THA performed with or without CT-based navigation in patients with ONFH. This study showed that use of the CT-based navigation system help prevent dislocation in patients with ONFH. 


\section{Conclusion}

CT-based navigation system provided a precise placement of components, and thus help prevent dislocation in patients with ONFH in the propensity score analysis.

\section{References}

Mont MA, Cherian JJ, Sierra RJ, Jones LC, Lieberman JR. (2015) Nontraumatic Osteonecrosis of the Femoral Head: Where Do We Stand Today? A Ten-Year Update. J Bone Joint Surg Am, 97(19), pp: 1604-27.

Johannson HR, Zywiel MG, Marker DR, Jones LC, McGrath MS, Mont MA. (2011) Osteonecrosis is not a predictor of poor outcomes in primary total hip arthroplasty: a systematic literature review. Int Orthop, 35(4), pp: 465-73.

Sugano N, Takao M, Sakai T, Nishii T, Miki H. (2012) Does CT-based navigation improve the longterm survival in ceramic-on-ceramic THA? Clin Orthop Relat Res, 470(11), pp: 3054-9.

Kobayashi S, Kubo T, Iwamoto Y, Fukushima W, Sugano N. (2018) Nationwide multicenter followup cohort study of hip arthroplasties performed for osteonecrosis of the femoral head. Int Orthop, 42(7), pp: $1661-8$.

Sugano N, Atsumi T, Ohzono K, Kubo T, Hotokebuchi T, Takaoka K. (2002) The 2001 revised criteria for diagnosis, classification, and staging of idiopathic osteonecrosis of the femoral head. J Orthop Sci, 7, pp: 601-5. 